\title{
SIMPLIFIED NEUTROSOPHIC INDETERMINATE DECISION MAKING METHOD WITH DECISION MAKERS' INDETERMINATE RANGES
}

\author{
Shigui DU ${ }^{1}$, Jun $\mathrm{YE}^{1,2^{*}}$, Rui YONG${ }^{2}$, Fangwei ZHANG ${ }^{1}$ \\ ${ }^{1}$ Institute of Rock Mechanics, Ningbo University, 315211 Ningbo, P. R. China \\ ${ }^{2}$ Department of Civil Engineering, Shaoxing University, 312000 Shaoxing, P. R. China
}

Received 27 January 2020; accepted 18 May 2020

\begin{abstract}
There exists the indeterminate situations of truth, falsity, indeterminacy degrees due to the uncertainty and inconsistency of decision makers' arguments in a complicated decision making (DM) problem. Then, existing neutrosophic set cannot describe the indeterminate information of truth, falsity, indeterminacy degrees. It is noted that the simplified neutrosophic set (SNS) is depicted by truth, falsity, indeterminacy degrees, while a neutrosophic number (NN) can be flexibly depicted by its determinate part and its indeterminate part. Regarding the indeterminate situations of truth, falsity, indeterminacy degrees in indeterminate DM problems, this study first presents a simplified neutrosophic indeterminate set (SNIS) to express the hybrid information of SNS and NN and defines the score, accuracy, and certainty functions of simplified neutrosophic indeterminate elements (SNIEs) with indeterminate ranges to compare SNIEs. Then, we introduce a SNIE weighted arithmetic averaging (SNIEWAA) operator and a SNIE weighted geometric averaging (SNIEWGA) operator to aggregate simplified neutrosophic indeterminate information. Next, a multi-attribute DM approach with decision makers' indeterminate ranges is established regarding the SNIEWAA and SNIEWGA operators in SNIS setting. Finally, the proposed DM approach is applied in a DM example on choosing a suitable slope design scheme to indicate the applicability and suitability of the proposed approach.
\end{abstract}

Keywords: simplified neutrosophic indeterminate set, simplified neutrosophic indeterminate element, simplified neutrosophic indeterminate element weighted arithmetic averaging (SNIEWAA) operator, simplified neutrosophic indeterminate element weighted geometric averaging (SNIEWGA) operator, decision making.

\section{Introduction}

In complicated decision making problems, the reasonable expression and aggregation of assessment information are two principal issues (Wu et al., 2019a, 2019b). To describe incomplete, indeterminate, and inconsistent information in the real world, a neutrosophic set (Smarandache, 1998) was proposed from the viewpoint of philosophy as a branch of neutrosophic theory and depicted independently by the truth, falsity, indeterminacy membership functions belonging to the subsets of the real standard interval $[0,1]$ or nonstandard interval $]^{-} 0,1^{+}[$. Based on the real standard interval $[0,1]$ in actual applications, Ye (2014a) introduced simplified neutrosophic sets (SNSs), implying single-valued neutrosophic sets (SvNSs) (Wang et al., 2010) and interval-valued neutrosophic sets (IvNSs) (Wang et al., 2005), as the subclass of the neutrosophic set, which is the generalization of fuzzy sets (Zadeh, 1965), intuitionistic fuzzy sets (IFSs) (Atanassov, 1986), and in- terval-valued IFSs (IvIFSs) (Atanassov \& Gargov, 1989), and then defined the operational relations and weighted aggregation operators of simplified neutrosophic elements (SNEs) for decision making (DM) applications. Since then, SNSs (SvNSs and IvNSs) have been wildly applied in DM (Liu \& Wang, 2014; Peng et al., 2016; Wu et al., 2016; Sahin \& Liu, 2017a, 2017b; Zhou et al., 2019; Köseoğlu et al., 2019), clustering analysis (Ye, 2014b), medical diagnosis (Thanh et al., 2017; Alia et al., 2018), control design (Gal et al., 2012; Can \& Ozguven, 2017), mechanical fault diagnosis (Ye, 2017), and so on.

As the further generalization of neutrosophic sets, refined neutrosophic sets (Smarandache, 2013a; Broumi \& Deli, 2014; Chen et al., 2017), neutrosophic multisets (Ye et al., 2015), and multivalued neutrosophic sets (Peng et al., 2015) were proposed and applied in DM and medical diagnosis problems. By combining neutrosophic sets

*Corresponding author. E-mails: yejun1@nbu.edu.cn; yehjun@aliyun.com 
with other fuzzy theories, some researchers proposed neutrosophic soft sets (Maji, 2013), interval neutrosophic rough sets (Broumi \& Smarandache, 2015), single-valued and interval neutrosophic hesitant fuzzy sets (Liu \& Shi, 2015), complex neutrosophic sets (Ali \& Smarandache, 2016), dynamical neutrosophic sets (Ye \& Fu, 2016; Thong et al., 2019), neutrosophic cubic set (Ali et al., 2016; Jun et al., 2017; Liu et al., 2019), normal neutrosophic sets (Şahin, 2018), single-valued neutrosophic 2-tuple linguistic sets (Wu et al., 2018), and their applications.

As another branch of neutrosophic theory, a neutrosophic number (NN) (Smarandache, 1998, 2013b, 2014) was proposed under indeterminate environment and represented as $e=a+\alpha I$ for $a, \alpha \in \mathfrak{R}$ and $I \in\left[I^{-}, I^{+}\right]$, where $a$ is a certain term and $\alpha I$ is an indeterminate term along with the indeterminate coefficient $\alpha$ and indeterminacy $I \in\left[I^{-}, I^{+}\right] . \mathrm{NN}$ indicates a family of interval numbers corresponding to different indeterminate ranges of $I \in\left[I^{-}\right.$, $I^{+}$, which demonstrates its flexibility and convenience in expressing indeterminate information. Therefore, NNs have been widely applied in many areas. For example, mechanical fault diagnosis (Ye, 2016), DM (P. D. Liu \& X. Liu, 2018), rock mechanics (Ye et al., 2017), optimization programming (Ye, 2018), and so on.

Then, there may exist the indeterminacy of the truth, falsity, indeterminacy degrees given by a group of decision makers due to the indeterminacy and inconsistency of decision makers' cognitions regarding object complexity and variability evaluated in the real DM problem. It is noted that the indeterminacy information of the truth, falsity, and indeterminacy degrees contains the hybrid information of SNS and NN, which cannot be expressed only by the neutrosophic set or NN. Since NN can flexibly depict such an indeterminacy with a changeable interval number $\left(e=\left[a+\alpha I^{-}, a+\alpha I^{+}\right]\right)$or a changeable single value $(e=$ $a+\alpha I)$ depending on specified indeterminate ranges of $I \in\left[I^{L}, I^{U}\right]$ or specified single values of $I \in\left[I^{L}, I^{U}\right]$, which shows its main highlight in an expression of indeterminate information. Then, SNS (IvSS and SvNS) can depict the truth, falsity, and indeterminacy degrees, but cannot depict such indeterminacy with a changeable interval number/single value of the truth/falsity/indeterminacy degree in indeterminate situations. Obviously, existing neutrosophic DM methods cannot handle such a DM problem with both the indeterminate information of the truth, falsity, indeterminacy arguments and the decision makers' indeterminate ranges/cognitions in indeterminate DM applications. If SNS is combined with NN based on an information expression advantage of both, we can present the new set concept and DM method based on the hybrid information of SNS and NN to carry out the aforementioned issues. Motivated by the new set concept and DM method, this study firstly proposes simplified neutrosophic indeterminate sets (SNISs) to express changeable IvNSs/ SvNSs corresponding to different indeterminate ranges/ values of $I \in\left[I^{-}, I^{+}\right]$and weighted aggregation operators of simplified neutrosophic indeterminate elements (SNIEs), and then establishes a multi-attribute DM method with decision makers' indeterminate ranges in indeterminate DM situations.

To the best of our knowledge, there exists no study regarding the proposed issues in existing literature. Hence, the main contributions of this study are: (1) to present SNIS and a ranking method of SNIEs, (2) to introduce a SNIE weighted arithmetic averaging (SNIEWAA) operator and a SNIE weighted geometric averaging (SNIEWGA) operator, (3) to establish a multi-attribute DM approach with decision makers' indeterminate ranges regarding the SNIEWAA and SNIEWGA operators in SNIS setting, and (4) to apply the proposed DM approach to an indeterminate DM example on choosing a suitable slope design scheme for an open pit mine in SNIS setting for indicating its flexibility and suitability under the indeterminate DM environment.

To realize this study, the rest of the article is constructed by the following parts. Section 1 introduces some preliminaries of SNSs and NNs. Section 2 presents a SNIS concept to depict the indeterminacy information of the truth, falsity, and indeterminacy degrees, and then defines the score, accuracy, and certainty functions of SNIEs with $I \in\left[I^{-}, I^{+}\right]$for ranking SNIEs. In Section 3, the SNIEWAA and SNIEWGA operators are proposed to aggregate SNIEs. For Section 4, a multi-attribute DM approach with decision makers' indeterminate ranges regarding the SNIEWAA and SNIEWGA operators is established in SNIS setting. Then, Section 5 applies the proposed DM approach to an indeterminate DM example on choosing a suitable slope design scheme for an open pit mine in SNIS setting for indicating its flexibility and effectiveness. Lastly, the conclusions and further research are indicated.

\section{Some preliminaries of SNSs and NNs}

As a subclass of a neutrosophic set (Smarandache, 1998), Ye (2014a) defined a SNS $S=\left\{\left\langle x_{k}, \tau_{S}\left(x_{k}\right), \cup_{S}\left(x_{k}\right)\right.\right.$, $\left.\left.\zeta_{S}\left(x_{k}\right)\right\rangle \mid x_{k} \in X\right\}$ in the universe set $X=\left\{x_{1}, x_{2}, \ldots, x_{n}\right\}$, where $\tau_{S}\left(x_{k}\right): X \rightarrow[0,1], v_{S}\left(x_{k}\right): X \rightarrow[0,1]$, and $\zeta_{S}\left(x_{k}\right)$ : $X \rightarrow[0,1](k=1,2, \ldots, n)$ are the truth, indeterminacy, and falsity membership degrees of the element $x_{k}$ to the set $S$, along with the condition $0 \leq \tau_{S}\left(x_{k}\right)+v_{S}\left(x_{k}\right)+\zeta_{S}\left(x_{k}\right) \leq 3$ for SvNS and $0 \leq \sup \tau_{S}\left(x_{k}\right)+\sup v_{S}\left(x_{k}\right)+\sup \zeta_{S}\left(x_{k}\right) \leq 3$ for IvNS and $x_{k} \in X$.

For the convenience of the representation, a component $\left\langle x_{k}, \tau_{S}\left(x_{k}\right), v_{S}\left(x_{k}\right), \zeta_{S}\left(x_{k}\right)\right\rangle$ in $S$ can be simply denoted as the simplified neutrosophic element (SNE) $s_{k}=\left\langle\tau_{k}, \mathrm{v}_{k}, \zeta_{k}\right\rangle$, which includes the interval-valued neutrosophic element (IvNE) $s_{k}=\left\langle\tau_{k}, v_{k}, \zeta_{k}\right\rangle=<\left[\tau_{k}^{-}, \tau_{k}^{+}\right],\left[v_{k}^{-}, v_{k}^{+}\right],\left[\zeta_{k}^{-}, \zeta_{k}^{+}\right]>$for $\tau_{k}, v_{k}, \zeta_{k} \subseteq[0,1]$ and single-valued neutrosophic element (SvNE) $s_{k}=<\tau_{k}, v_{k}, \zeta_{k}>$ for $\tau_{k}, v_{k}, \zeta_{k} \in[0,1]$.

Set two SNEs as $\left.s_{1}=<\tau_{1}, v_{1}, \zeta_{1}\right\rangle$ and $s_{2}=<\tau_{2}, v_{2}$, $\zeta_{2}>$ and $\omega>0$. Then, there exist the following relations (Smarandache, 1998; Wang et al., 2005; Ye, 2014a; Zhang et al., 2014):

1) $s_{1} \subseteq s_{2} \Leftrightarrow \tau_{1}^{-} \leq \tau_{2}^{-}, \tau_{1}^{+} \leq \tau_{2}^{+}, v_{1}^{-} \geq v_{2}^{-}, v_{1}^{+} \geq v_{2}^{+}$, $\zeta_{1}^{-} \geq \zeta_{2}^{-}, \zeta_{1}^{+} \geq \zeta_{2}^{+}$for IvNEs and $\tau_{1} \leq \tau_{2}, v_{1} \geq v_{2}$, $\zeta_{1} \geq \zeta_{2}$ for SvNEs; 
2) $s_{1}=s_{2} \Leftrightarrow s_{1} \subseteq s_{2}$ and $s_{2} \subseteq s_{1}$;

3) (Complement of $\left.s_{1}\right)\left(s_{1}\right)^{C}=<\left[\zeta_{1}^{-}, \zeta_{1}^{+}\right],\left[1-v_{1}^{+}, 1-v_{1}^{-}\right]$, $\left[\tau_{1}^{-}, \tau_{1}^{+}\right]>$for IvNE and $\left(s_{1}\right)^{\mathrm{C}}=\left\langle\zeta_{1}, 1-v_{1}, \tau_{1}>\right.$ for SvNE;

4) $s_{1} \oplus s_{2}=\left\langle\left[\tau_{1}^{-}+\tau_{2}^{-}-\tau_{1}^{-} \tau_{2}^{-}, \tau_{1}^{+}+\tau_{2}^{+}-\tau_{1}^{+} \tau_{2}^{+}\right]\right.$,

$\left.\left[v_{1}^{-} v_{2}^{-}, v_{1}^{+} v_{2}^{+}\right],\left[\zeta_{1}^{-} \zeta_{2}^{-}, \zeta_{1}^{+} \zeta_{2}^{+}\right]\right\rangle$for IvNEs and $s_{1} \oplus s_{2}=$ $\left\langle\tau_{1}+\tau_{2}-\tau_{1} \tau_{2}, v_{1} v_{2}, \zeta_{1} \zeta_{2}\right\rangle$ for SvNEs;

5) $s_{1} \otimes s_{2}=\left\langle\left[\tau_{1}^{-} \tau_{2}^{-}, \tau_{1}^{+} \tau_{2}^{+}\right],\left[v_{1}^{-}+v_{2}^{-}-v_{1}^{-} v_{2}^{-}, v_{1}^{+}+v_{2}^{+}-\right.\right.$ $\left.\left.v_{1}^{+} v_{2}^{+}\right],\left[\zeta_{1}^{-}+\zeta_{2}^{-}-\zeta_{1}^{-} \zeta_{2}^{-}, \zeta_{1}^{+}+\zeta_{2}^{+}-\zeta_{1}^{+} \zeta_{2}^{+}\right]\right\rangle$for IvNEs and $s_{1} \otimes s_{2}=\left\langle\tau_{1} \tau_{2}, v_{1}+v_{2}-v_{1} v_{2}, \zeta_{1}+\zeta_{2}-\zeta_{1} \zeta_{2}\right\rangle$ for SvNEs;

6) $\omega s_{1}=\left\langle\left[1-\left(1-\tau_{1}^{-}\right)^{\omega}, 1-\left(1-\tau_{1}^{+}\right)^{\omega}\right],\left[\left(v_{1}^{-}\right)^{\omega},\left(v_{1}^{+}\right)^{\omega}\right]\right.$, $\left.\left[\left(\zeta_{1}^{-}\right)^{\omega},\left(\zeta_{1}^{+}\right)^{\omega}\right]\right\rangle$ for IvNE and $\omega s_{1}=\left\langle 1-\left(1-\tau_{1}\right)^{\omega}\right.$, $\left.v_{1}^{\omega}, \zeta_{1}^{\omega}\right\rangle$ for SvNE;

7) $s_{1}^{\omega}=\left\langle\left[\left(\tau_{1}^{-}\right)^{\omega},\left(\tau_{1}^{+}\right)^{\omega}\right],\left[1-\left(1-v_{1}^{-}\right)^{\omega}, 1-\left(1-v_{1}^{+}\right)^{\omega}\right]\right.$,

$\left.\left[1-\left(1-\zeta_{1}^{-}\right)^{\omega}, 1-\left(1-\zeta_{1}^{+}\right)^{\omega}\right]\right\rangle$ for IvNE and $s_{1}^{\omega}=\left\langle\tau_{1}^{\omega}\right.$, $\left.1-\left(1-v_{1}\right)^{\omega}, 1-\left(1-\zeta_{1}\right)^{\omega}\right\rangle$ for SvNE.

Set $s_{k}=\left\langle\tau_{k}, v_{k}, \zeta_{k}\right\rangle(k=1,2, \ldots, n)$ as a group of SNEs. Then the SvNE weighted arithmetic averaging (SvNEWAA), IvNE weighted arithmetic averaging (IvNEWAA), SvNE weighted geometric averaging (SvNEWGA), and IvNE weighted geometric averaging (IvNEWGA) operators defined in Zhang et al. (2014) and Peng et al. (2016) are introduced, respectively, below:

$$
\begin{aligned}
& \operatorname{SvNEWAA}\left(s_{1}, s_{2}, \ldots, s_{n}\right)=\sum_{k=1}^{n} \omega_{k} s_{k}=\left\langle 1-\prod_{k=1}^{n}\left(1-\tau_{k}\right)^{\omega_{k}},\right. \\
& \left.\prod_{k=1}^{n}\left(v_{k}\right)^{\omega_{k}}, \prod_{k=1}^{n}\left(\zeta_{k}\right)^{\omega_{k}}\right\rangle \text { for SvNEs; } \\
& \operatorname{IvNEWAA}\left(s_{1}, s_{2}, \ldots, s_{n}\right)=\sum_{k=1}^{n} \omega_{k} s_{k}= \\
& \left\langle\left[1-\prod_{k=1}^{n}\left(1-\tau_{k}^{-}\right)^{\omega_{k}}, 1-\prod_{k=1}^{n}\left(1-\tau_{k}^{+}\right)^{\omega_{k}}\right],\left[\prod_{k=1}^{n}\left(v_{k}^{-}\right)^{\omega_{k}},\right.\right. \\
& \left.\left.\prod_{k=1}^{n}\left(v_{k}^{+}\right)^{\omega_{k}}\right],\left[\prod_{k=1}^{n}\left(\zeta_{k}^{-}\right)^{\omega_{k}}, \prod_{k=1}^{n}\left(\zeta_{k}^{+}\right)^{\omega_{k}}\right]\right\rangle \text { for IvNEs; (2) } \\
& \operatorname{SvNEWGA}\left(s_{1}, s_{2}, \ldots, s_{n}\right)=\prod_{k=1}^{n} s_{k}^{\omega_{k}}=\left\langle\prod_{k=1}^{n}\left(\tau_{k}\right)^{\omega_{k}},\right. \\
& \left.1-\prod_{k=1}^{n}\left(1-v_{k}\right)^{\omega_{k}}, 1-\prod_{k=1}^{n}\left(1-\zeta_{k}\right)^{\omega_{k}}\right\rangle \text { for SvNEs; } \\
& \operatorname{IvNEWGA}\left(s_{1}, s_{2}, \ldots, s_{n}\right)=\prod_{k=1}^{n} s_{k}^{\omega_{k}}= \\
& \left\langle\left[\prod_{k=1}^{n}\left(\tau_{k}^{-}\right)^{\omega_{k}}, \prod_{k=1}^{n}\left(\tau_{k}^{+}\right)^{\omega_{k}}\right],\left[1-\prod_{k=1}^{n}\left(1-\mathrm{v}_{k}^{-}\right)^{\omega_{k}},\right.\right. \\
& \left.1-\prod_{k=1}^{n}\left(1-v_{k}^{+}\right)^{\omega_{k}}\right],\left[1-\prod_{k=1}^{n}\left(1-\zeta_{k}^{-}\right)^{\omega_{k}}\right. \text {, }
\end{aligned}
$$

\section{SNISs and ranking method}

Based on the hybrid concept of both SNS and NN, we can give the definition of a SNIS as the generalization of a SNS concept in indeterminate and inconsistent situations.

Definition 1. Set $X=\left\{x_{1}, x_{2}, \ldots, x_{n}\right\}$ as a universe set. A SNIS $Z$ is defined as the following expression:

$$
Z=\left\{\left\langle x_{k}, \tau_{Z}\left(x_{k}, I\right), \mathrm{v}_{Z}\left(x_{k}, I\right), \zeta_{Z}\left(x_{k}, I\right)\right\rangle \mid x_{k} \in X\right\},
$$

where $\tau_{Z}\left(x_{k}, I\right)=a_{k}+\alpha_{k} I \subseteq[0,1], v_{Z}\left(x_{k}, I\right)=b_{k}+\beta_{k} I \subseteq$ $[0,1]$, and $\zeta_{Z}\left(x_{k}, I\right)=c_{k}+\gamma_{k} I \subseteq[0,1]$ for $x_{k} \in X(k=1,2$, .., $n)$ and $I \in\left[I^{-}, I^{+}\right]$are the truth NN, the indeterminacy $\mathrm{NN}$, and the falsity NN, respectively, along with the condition $0 \leq \sup \tau_{Z}\left(x_{k}, I\right)+\operatorname{supv} v_{Z}\left(x_{k}, I\right)+\sup \zeta_{Z}\left(x_{k}, I\right) \leq 3$.

Then, the basic component $\left\langle x_{k}, \tau_{Z}\left(x_{k}, I\right), \cup_{Z}\left(x_{k}, I\right)\right.$, $\left.\zeta_{Z}\left(x_{k}, I\right)\right\rangle$ in a SNIS $Z$ for $x_{k} \in X(k=1,2, \ldots, n)$ and $I \in\left[I^{-}, I^{+}\right]$is simply denoted as $z_{k}=\left\langle\tau_{k}(I), \mathrm{v}_{k}(I)\right.$, $\left.\zeta_{k}(I)\right\rangle=\left\langle a_{k}+\alpha_{k} I, b_{k}+\beta_{k} I, c_{k}+\gamma_{k} I\right\rangle$, which is named SNIE. 
Regarding the different indeterminate ranges/values of $I \in\left[I^{-}, I^{+}\right]$, a SNIS Z can consist of the SNS family. Especially when the indeterminate parts $\alpha_{k} I, \beta_{k} I, \gamma_{k} I$ in $Z$ are all single values or interval numbers, the SNIS $Z$ reduces to the SvNS or IvNS family as the special case of the SNIS $Z$. For example, let a SNIS be $Z=\left\{\mathrm{z}_{1}, \mathrm{z}_{2}\right\}=\{<0.6+0.2 I$, $0.1+0.1 I, 0.2+0.1 I>,<0.7+0.1 I, 0.1+0.2 I, 0.2+0.1 I\rangle$ for $I \in\left[I^{-}, I^{+}\right]=[0,1]$. If $I=[0,0.2],[0,0.6],[0,1]$ or $I=0,0.2,0.6,1$ are specified, then there are the following SvNS family or IvNS family:

$$
Z= \begin{cases}\{<0.6,0.1,0.2>, \quad<0.7,0.1,0.2>\} & \text { for } I=0, \\ \{<0.64,0.12,0.22>,<0.72,0.14,0.22>\} & \text { for } I=0.2, \\ \{<0.72,0.16,0.26>,<0.76,0.22,0.26>\} & \text { for } I=0.6, \\ \{<0.8,0.2,0.3>, \quad<0.8,0.3,0.3>\} & \text { for } I=1 .\end{cases}
$$

Or

$$
\begin{aligned}
& Z=\left\{\begin{array}{l}
\{<[0.6,0.64],[0.1,0.12],[0.2,0.22]>, \\
\{<[0.6,0.72],[0.1,0.16],[0.2,0.26]>, \\
\{<[0.6,0.8],[0.1,0.2],[0.2,0.3]>,
\end{array}\right. \\
& <[0.7,0.72],[0.1,0.14],[0.2,0.22]>\} \text { for } I=[0,0.2], \\
& <[0.7,0.76],[0.1,0.22],[0.2,0.26]>\} \text { for } I=[0,0.6], \\
& <[0.7,0.8],[0.1,0.3],[0.2,0.3]>\} \quad \text { for } I=[0,1] .
\end{aligned}
$$

Obviously, SNIS shows the advantages of its convenience and flexibility in the indeterminate information expressions regarding different indeterminate ranges/values of $I \in\left[I^{-}, I^{+}\right]$.

To compare two SNIEs, we need to define the score, accuracy and certainty functions of SNIE with $I \in\left[I^{-}, I^{+}\right]$ and their ranking method with $I \in\left[I^{-}, I^{+}\right]$below.

Definition 2. Set $z=\langle\tau(I), \mathcal{v}(I), \zeta(I)\rangle=\langle a+\alpha I, b+\beta I, c+\gamma I\rangle$ for $I \in\left[I^{-}, I^{+}\right]$as any SNIE, then its score, accuracy and certainty functions with $I \in\left[I^{-}, I^{+}\right]$can be defined, respectively, as the following formulae:

$$
\begin{aligned}
& S(z, I)=\{4+\inf \tau(I)+\sup \tau(I)-\inf v(I)-\sup v(I)- \\
& \inf \zeta(I)-\sup \zeta(I)\} / 6=\left\{4+\left[2 a+\alpha\left(I^{-}+I^{+}\right)\right]-\right. \\
& \left.\left[2 b+\beta\left(I^{-}+I^{+}\right)\right]-\left[2 b+\beta\left(I^{-}+I^{+}\right)\right]\right\} / 6, \\
& S(z, I) \in[0,1] ; \\
& H(z, I)=\{\inf \tau(I)+\sup \tau(I)-\inf \zeta(I)-\sup \zeta(I)\} / 2= \\
& \left\{2 a+\alpha\left(I^{-}+I^{+}\right)-\left[2 c+\gamma\left(I^{-}+I^{+}\right)\right]\right\} / 2, \\
& H(z, I) \in[-1,1] ; \\
& D(z, I)=[\inf \tau(I)+\sup \tau(I)] / 2=\left[2 a+\alpha\left(I^{-}+I^{+}\right)\right] / 2, \\
& D(z, I) \in[0,1] .
\end{aligned}
$$

By the three functions $S(z, I), H(z, I)$ and $D(z, I)$, the ranking method of SNIEs is presented by the following definition.

Definition 3. Let $z_{i}=\left\langle\tau_{i}(I), \cup_{i}(I), \zeta_{i}(I)\right\rangle=\left\langle a_{i}+\alpha_{i} I, b_{i}+\right.$ $\left.\beta_{i} I, c_{i}+\gamma_{i} I\right\rangle(i=1,2)$ for $I \in\left[I^{-}, I^{+}\right]$be SNIEs. Then, their ranking method with $I \in\left[I^{-}, I^{+}\right]$can be presented as follows:

1) $z_{1}>z_{2}$ for $S\left(z_{1}, I\right)>S\left(z_{2}, I\right)$;

2) $z_{1}>z_{2}$ for $S\left(z_{1}, I\right)=S\left(z_{2}, I\right)$ and $H\left(z_{1}, I\right)>H\left(z_{2}, I\right)$;
3) $z_{1}>z_{2}$ for $S\left(z_{1}, I\right)=S\left(z_{2}, I\right), H\left(z_{1}, I\right)=H\left(z_{2}, I\right)$ and $D\left(z_{1}, I\right)>D\left(z_{2}, I\right)$

4) $z_{1}=z_{2}$ for $S\left(z_{1}, I\right)=S\left(z_{2}, I\right), H\left(z_{1}, I\right)=H\left(z_{2}, I\right)$ and $D\left(z_{1}, I\right)=D\left(z_{2}, I\right)$.

\section{Weighted aggregation operators of SNIEs}

Based on the aggregation operators of Eqns (1)-(4) (Zhang et al., 2014; Peng et al., 2016) and the above NN operational relations, we can extend them to the two weighted aggregation operators of SNIEs in this section.

Theorem 1. Set $z_{k}=\left\langle\tau_{k}(I), \cup_{k}(I), \zeta_{k}(I)\right\rangle=\left\langle a_{k}+\alpha_{k} I, b_{k}+\right.$ $\left.\beta_{k} I, c_{k}+\gamma_{k} I\right\rangle$ for $I \in\left[I^{-}, I^{+}\right](k=1,2, \ldots, n)$ as a group of SNIEs. Based on the SvNEWAA and IvNEWAA operators (Zhang et al., 2014; Peng et al., 2016), the aggregated SNIE is given by the SNIEWAA operator:

$$
\begin{aligned}
& \operatorname{SNIEWAA}\left(z_{1}, z_{2}, \cdots, z_{n}\right)=\sum_{k=1}^{n} \omega_{k} z_{k}= \\
& \left\langle 1-\prod_{k=1}^{n}\left(1-a_{k}-\alpha_{k} I\right)^{\omega_{k}}, \prod_{k=1}^{n}\left(b_{k}+\beta_{k} I\right)^{\omega_{k}},\right. \\
& \left.\prod_{k=1}^{n}\left(c_{k}+\gamma_{k} I\right)^{\omega_{k}}\right\rangle
\end{aligned}
$$

where $\omega_{k} \in[0,1](k=1,2, \ldots, n)$ is the weight of $x_{k}$ for $\sum_{k=1}^{n} \omega_{k}=1$.

Theorem 2. Set $z_{k}=\left\langle\tau_{k}(I), \mathrm{v}_{k}(I), \zeta_{k}(I)\right\rangle=\left\langle a_{k}+\alpha_{k} I, b_{k}+\right.$ $\left.\beta_{k} I, c_{k}+\gamma_{k} I\right\rangle$ for $I \in\left[I^{-}, I^{+}\right](k=1,2, \ldots, n)$ as a group of SNIEs. Based on the SvNEWGA and IvNEWGA operators (Zhang et al., 2014; Peng et al., 2016), the aggregated SNIE is given by the SNIEWGA operator:

$$
\begin{aligned}
& \operatorname{SNIEWGA}\left(z_{1}, z_{2}, \cdots, z_{n}\right)=\prod_{k=1}^{n} z_{k}^{\omega_{k}}= \\
& \left\langle\prod_{k=1}^{n}\left(a_{k}+\alpha_{k} I\right)^{\omega_{k}}, 1-\prod_{k=1}^{n}\left(1-b_{k}-\beta_{k} I\right)^{\omega_{k}},\right. \\
& \left.1-\prod_{k=1}^{n}\left(1-c_{k}-\gamma_{k} I\right)^{\omega_{k}}\right\rangle,
\end{aligned}
$$

where $\omega_{k} \in[0,1](k=1,2, \ldots, n)$ is the weight of $x_{k}$ for $\sum_{k=1}^{n} \omega_{k}=1$.

Clearly, Eqns (8) and (9) contain Eqns (1)-(4) corresponding to different indeterminate values and ranges of $I \in\left[I^{-}, I^{+}\right]$. Especially when some single value $I=$ $I^{-}=I^{+}$or some interval value $I=\left[I^{-}, I^{+}\right]$, the SNIEWAA and SNIEWGA operators reduce to the SvNEWAA and SvNEWGA operators or the IvNEWAA and IvNEWGA operators as the special cases of the SNIEWAA and SNIEWGA operators.

As for the properties of the SvNEWAA, IvNEWAA, SvNEWGA, and IvNEWGA operators (Zhang et al., 2014; Peng et al., 2016), it is obvious that the SNIEWAA and 
SNIEWGA operators also imply the following properties:

1) Idempotency: Set $z_{k}=\left\langle\tau_{k}(I), \cup_{k}(I), \zeta_{k}(I)\right\rangle=\left\langle a_{k}+\right.$ $\left.\alpha_{k} I, b_{k}+\beta_{k} I, c_{k}+\gamma_{k} I\right\rangle$ for $I \in\left[I^{-}, I^{+}\right](k=1,2$, $\ldots, n)$ as a group of SNIEs. If $z_{k}=z$ for $k=1$, $2, \ldots, n$, then $\operatorname{SNIEWAA}\left(z_{1}, z_{2}, \cdots, z_{n}\right)=z$ and $\operatorname{SNIEWGA}\left(z_{1}, z_{2}, \cdots, z_{n}\right)=z$ exist.

2) Boundedness: Set $z_{k}=\left\langle\tau_{k}(I), v_{k}(I), \zeta_{k}(I)\right\rangle=\left\langle a_{k}+\right.$ $\left.\alpha_{k} I, b_{k}+\beta_{k} I, c_{k}+\gamma_{k} I\right\rangle$ for $I \in\left[I^{-}, I^{+}\right](k=1,2, \ldots$, $n$ ) as a group of SNIEs and let

$$
\begin{aligned}
& z_{\min }=\left\{\begin{array}{l}
{\left[\min _{k}\left(a_{k}+\alpha_{k} I^{-}\right), \min _{k}\left(a_{k}+\alpha_{k} I^{+}\right)\right],} \\
{\left[\max _{k}\left(c_{k}+\gamma_{k} I^{-}\right), \max _{k}\left(c_{k}+\gamma_{k} I^{+}\right)\right]}
\end{array}\right. \\
& \left.\left[\max _{k}\left(b_{k}+\beta_{k} I^{-}\right), \max _{k}\left(b_{k}+\beta_{k} I^{+}\right)\right],\right\rangle, \\
& z_{\max }=\left\{\begin{array}{l}
{\left[\max _{k}\left(a_{k}+\alpha_{k} I^{-}\right), \max _{k}\left(a_{k}+\alpha_{k} I^{+}\right)\right]} \\
{\left[\min _{k}\left(c_{k}+\gamma_{k} I^{-}\right), \min _{k}\left(c_{k}+\gamma_{k} I^{+}\right)\right]}
\end{array}\right] \\
& \left.\left[\min _{k}\left(b_{k}+\beta_{k} I^{-}\right), \min _{k}\left(b_{k}+\beta_{k} I^{+}\right)\right],\right\rangle \text {. }
\end{aligned}
$$

Then, there are $z_{\min } \leq \operatorname{SNIEWAA}\left(z_{1}, z_{2}, \cdots, z_{n}\right) \leq$ $z_{\max }$ and $z_{\min } \leq \operatorname{SNIEWGA}\left(z_{1}, z_{2}, \cdots, z_{n}\right) \leq z_{\max }$.

3) Monotonicity: Set $z_{k}=\left\langle\tau_{k}(I), v_{k}(I), \zeta_{k}(I)\right\rangle$ and $z_{k}^{*}=\left\langle\tau_{k}^{*}(I), v_{k}^{*}(I), \zeta_{k}^{*}(I)\right\rangle$ for $I \in\left[I^{-}, I^{+}\right] \quad(k=1$, $2, \ldots, n)$ as two groups of SNIEs. If $z_{k} \subseteq z_{k}^{*}$, then $\operatorname{SNIEWAA}\left(z_{1}, z_{2}, \cdots, z_{n}\right) \subseteq \operatorname{SNIEWAA}\left(z_{1}^{*}, z_{2}^{*}, \cdots, z_{n}^{*}\right)$ and $\operatorname{SNIEWGA}\left(z_{1}, z_{2}, \cdots, z_{n}\right) \subseteq \operatorname{SNIEWGA}\left(z_{1}^{*}, z_{2}^{*}\right.$, $\cdots, z_{n}^{*}$ ) can hold.

\section{DM method with decision makers' indeterminate ranges}

This section proposes a multi-attribute DM method with decision makers' indeterminate ranges regarding the SNIEWAA and SNIEWGA operators and the ranking method in SNIS setting.

Assume that there exists a multi-attribute DM problem containing a set of $m$ alternatives $M=\left\{M_{1}, M_{2}, \ldots, M_{m}\right\}$ and a set of $n$ attributes $R=\left\{R_{1}, R_{2}, \ldots, R_{n}\right\}$. Then the weigh vector of $R$ is specified by $\omega=\left(\omega_{1}, \omega_{2}, \ldots, \omega_{n}\right)$. Thus, the alternatives $M_{j}(j=1,2, \ldots, m)$ are satisfactorily assessed over the attributes $R_{k}(k=1,2, \ldots, n)$ by the SNIE $z_{j k}=\left\langle\tau_{j k}(I), \cup_{j k}(I), \zeta_{j k}(I)\right\rangle=\left\langle a_{j k}+\alpha_{j k} I, b_{j k}+\beta_{j k} I, c_{j k}+\right.$ $\left.\gamma_{j k} I\right\rangle$ for $a_{j k}+\alpha_{j k} I \subseteq[0,1], b_{j k}+\beta_{j k} I \subseteq[0,1], c_{j k}+\gamma_{j k} I \subseteq$ $[0,1]$, and $I \in\left[I^{-}, I^{+}\right](k=1,2, \ldots, n ; j=1,2, \ldots, m)$. Hence, all the SNIEs specified by decision makers can be constructed as the SNIE decision matrix $Z=\left(z_{j k}\right)_{m \times n}$.

Corresponding to the aggregation operator of Eqns (8) or (9) and the ranking method, we present a multiattribute DM method with decision makers' indeterminate ranges for solving indeterminate DM problems with SNIS information and give the following decision steps:

Step 1: Based on Eqns (8) or (9) regarding the indeterminate range of $I \in\left[I^{-}, I^{+}\right]$specified by the decision makers' indeterminate degrees, the aggregation value of SNIEs $z_{j k}$ for $M_{j}(j=1,2, \ldots, m)$ is calculated by using the following aggregation operator:

$$
\begin{aligned}
& z_{j}=\operatorname{SNIEWAA}\left(z_{j 1}, z_{j 2}, \cdots, z_{j n}\right)=\sum_{k=1}^{n} \omega_{k} z_{j k}= \\
& \left\langle\left[1-\prod_{k=1}^{n}\left(1-a_{j k}-\alpha_{j k} I\right)^{\omega_{k}}\right], \prod_{k=1}^{n}\left(b_{j k}+\beta_{j k} I\right)^{\omega_{k}},\right. \\
& \left.\prod_{k=1}^{n}\left(c_{j k}+\gamma_{j k} I\right)^{\omega_{k}}\right\rangle ;
\end{aligned}
$$

or

$$
\begin{aligned}
& z_{j}=\operatorname{SNIEWGA}\left(z_{j 1}, z_{j 2}, \cdots, z_{j n}\right)=\prod_{k=1}^{n} z_{j k}^{\omega_{k}}= \\
& \left\langle\prod_{k=1}^{n}\left(a_{j k}+\alpha_{j k} I\right)^{\omega_{k}}, 1-\prod_{k=1}^{n}\left(1-b_{j k}-\beta_{j k} I\right)^{\omega_{k}},\right. \\
& \left.1-\prod_{k=1}^{n}\left(1-c_{j k}-\gamma_{j k} I\right)^{\omega_{k}}\right\rangle .
\end{aligned}
$$

Step 2: The values of the score function $S\left(z_{j}, I\right)\left(H\left(z_{j}, I\right)\right.$ and $D\left(z_{j}, I\right)$ if necessary) are calculated by Eqn (5) (Eqns (6) and (7)).

Step 3: The alternatives are ranked based on the ranking method in Definition 3 and the best one is selected.

Step 4: End.

\section{Indeterminate DM example on choosing a suitable open pit mine slope design scheme}

Open pit mine slope design is a fundamental issue in the process of mine design and operation to provide an optimal excavation configuration in the context of safety, ore recovery and financial return (Read \& Stacey, 2009). Hence, investors and operators firstly ensure the open pit mine slope stability for preventing the potential risks caused by slope failure (Yong et al., 2019). Then, the economic benefit of mining needs to be considered and ore recovery must be maximized to meet the economic needs of owners. Moreover, open pit mines in most countries generally have mining regulations that specify environmental requirements. It is obvious that the safety, economic and environmental factors should be considered as main assessment indices in the open pit mine slope design.

Let us consider a multi-attribute DM problem on choosing a suitable slope design scheme (alternative) for an open pit mine. Suppose that there is a set of four potential alternatives $M=\left\{M_{1}, M_{2}, M_{3}, M_{4}\right\}$ for the open pit mine, which must be satisfactorily assessed by the three 
indices (attributes): the safety factor $\left(R_{1}\right)$, the economic factor $\left(R_{2}\right)$, and the environmental factor $\left(R_{3}\right)$. Then the weight vector of the three attributes is specified as $\omega=$ $(0.36,0.3,0.34)$ by experts/decision makers.

Then, experts/decision makers are required to give the satisfactory assessment of each alternative $M_{j}(j=$ $1,2,3,4)$ over the attributes $R_{k}(k=1,2,3)$ by the assessment information of the truth, falsity, and indeterminacy NNs $a_{j k}+\alpha_{j k} I \subseteq[0,1], \quad b_{j k}+\beta_{j k} I \subseteq[0,1]$, and $c_{j k}+\gamma_{j k} I \subseteq[0,1]$ for the specified indeterminacy $I \in[0,1.5]$, which can be constructed as SNIEs $z_{j k}=\left\langle\tau_{j k}(I), \mathrm{v}_{j k}(I), \zeta_{j k}(I)\right\rangle=\left\langle a_{j k}+\alpha_{j k} I, b_{j k}+\beta_{j k} I, c_{j k}+\right.$ $\left.\gamma_{j k} I\right\rangle(k=1,2,3 ; j=1,2,3,4)$ and their decision matrix:

$$
\begin{aligned}
& Z=\left[\begin{array}{l}
<0.7+0.2 I, 0.1+0.3 I, 0.1+0.1 I> \\
<0.8+0.1 I, 0.1+0.2 I, 0.1+0.3 I> \\
<0.7+0.1 I, 0.2+0.1 I, 0.1+0.2 I> \\
<0.8+0.1 I, 0.1+0.2 I, 0.2+0.1 I>
\end{array}\right. \\
& <0.7+0.2 I, 0.2+0.1 I, 0.2+0.2 I> \\
& <0.7+0.2 I, 0.2+0.1 I, 0.3+0.1 I> \\
& <0.8+0.1 I, 0.2+0.1 I, 0.1+0.2 I> \\
& <0.7+0.1 I, 0.1+0.2 I, 0.2+0.1 I> \\
& <0.6+0.2 I, 0.2+0.2 I, 0.2+0.2 I> \\
& <0.7+0.1 I, 0.2+0.2 I, 0.1+0.1 I> \\
& <0.7+0.2 I, 0.3+0.1 I, 0.2+0.1 I> \\
& <0.7+0.1 I, 0.2+0.1 I, 0.2+0.2 I>]
\end{aligned}
$$

Thus, the developed approach is utilized for the indeterminate DM problem with $I \in[0,1.5]$ and described by the following decision process:

First, the aggregation values of SNIEs $z_{j k}$ for $M_{j}(j=1$, $2,3,4)$ are calculated by Eqns (10) or (11) for the specified indeterminacies $I=\left[I^{-}, I^{+}\right]=[0,0],[0,0.5],[0,1],[0,1.5]$ and tabulated in Table 1 and Table 2.

Then, the values of the score function $S\left(z_{j}, I\right)$ are calculated by Eqn (5). Consequently, all the decision results regarding the SNIEWAA and SNIEWGA operators are shown in Table 3 and Table 4, respectively.

Table 1. Aggregated values of the SNIEWAA operator

\begin{tabular}{|c|c|}
\hline$I=\left[I^{-}, I^{+}\right]$ & Aggregated value \\
\hline \multirow{5}{*}{$I=[0,0]$} & $z_{1}=<[0.6692,0.6692],[0.1558,0.1558],[0.1558,0.1558]>$, \\
& $z_{2}=<[0.7407,0.7407],[0.1558,0.1558],[0.1390,0.1390]>$, \\
& $\left.z_{3}=<[0.7344,0.7344],[0.2296,0.2296],[0.1266,0.1266]\right\rangle$, \\
& $z_{4}=<[0.7407,0.7407],[0.1266,0.1266],[0.2000,0.2000]>$ \\
\hline \multirow{5}{*}{$I=[0,0.5]$} & $z_{1}=<[0.6692,0.7704],[0.1558,0.2660],[0.1558,0.2337]>$, \\
& $z_{2}=<[0.7407,0.8055],[0.1558,0.2455],[0.1390,0.2325]>$, \\
& $z_{3}=<[0.7344,0.8012],[0.2296,0.2803],[0.1266,0.2158]>$, \\
& $z_{4}=<[0.7407,0.7920],[0.1266,0.2158],[0.2000,0.2660]>$ \\
\hline \multirow{5}{*}{$I=[0,1]$} & $z_{1}=<[0.6692,0.8734],[0.1558,0.3669],[0.1558,0.3117]>$, \\
& $z_{2}=<[0.7407,0.8734],[0.1558,0.3308],[0.1390,0.3160]>$, \\
& $z_{3}=<[0.7344,0.8717],[0.2296,0.3308],[0.1266,0.3000]>$, \\
& $z_{4}=<[0.7407,0.8442],[0.1266,0.3000],[0.2000,0.3308]>$ \\
\hline \multirow{5}{*}{$I=[0,1.5]$} & $z_{1}=<[0.6692,1.0000],[0.1558,0.4649],[0.1558,0.3896]>$, \\
& $z_{2}=<[0.7407,1.0000],[0.1558,0.4146],[0.1390,0.3961]>$, \\
& $z_{3}=<[0.7344,1.0000],[0.2296,0.3812],[0.1266,0.3822]>$, \\
& $z_{4}=<[0.7407,0.8990],[0.1266,0.3822],[0.2000,0.3951]>$. \\
\hline
\end{tabular}

In Tables 1 and 2, the ranking orders of alternatives and the best slope design schemes regarding the SNIEWAA and SNIEWGA operators are identical when the indeterminate ranges are $I=[0,0],[0,0.5]$, while the ranking orders and the best ones regarding the SNIEWAA and SNIEWGA operators indicate some difference when the indeterminate ranges are $I=[0,1],[0,1.5]$. Clearly, the different indeterminate ranges can affect the ranking orders of alternatives. Then the final decision result depends on the indeterminate range of $I \in\left[I^{-}, I^{+}\right]$specified by the decision makers, which demonstrate the effectiveness and flexibility of the proposed DM method in simplified neutrosophic indeterminate setting.

Especially when $I=[0,0]=0$ in Tables 1 and 2, the proposed simplified neutrosophic indeterminate DM method is reduced to the DM methods based on the SvNEWAA and SvNEWGA operators (Zhang et al., 2014; Peng et al., 2016), while when $I=[0,0.5],[0,1],[0,1.5]$ in Tables 1 and 2, the proposed simplified neutrosophic indeterminate DM method is reduced to the DM methods based on the IvNEWAA and IvNEWGA operators (Zhang et al., 2014; Peng et al., 2016). Obviously, the proposed simplified neutrosophic indeterminate DM method contains single-valued and interval neutrosophic DM methods (Zhang et al., 2014; Peng et al., 2016) because SNIS contains its SNS family (SvNS family or IvNS family) depending on the indeterminate values/ranges of $I \in\left[I^{-}, I^{+}\right]$. Therefore, the proposed DM method is the generalization of existing simplified neutrosophic DM methods (Zhang et al., 2014; Peng et al., 2016), while existing simplified neutrosophic DM methods (Zhang et al., 2014; Peng et al., 2016) are only the special cases of the proposed DM method with the specified indeterminate value/range of $I$ $\in\left[I^{-}, I^{+}\right]$. Since the proposed DM method indicates the advantage of its flexibility and generalization by comparison with existing simplified neutrosophic DM methods (Zhang et al., 2014; Peng et al., 2016), the proposed DM method is superior to existing ones (Zhang et al., 2014; Peng et al., 2016).

Table 2. Aggregated values of the SNIEWGA operator

\begin{tabular}{|c|c|}
\hline$I=\left[I^{-}, I^{+}\right]$ & Aggregated value \\
\hline \multirow{5}{*}{$I=[0,0]$} & $z_{1}=<[0.6643,0.6643],[0.1653,0.1653],[0.1653,0.1653]>$, \\
& $z_{2}=<[0.7345,0.7345],[0.1653,0.1653],[0.1654,0.1654]>$, \\
& $z_{3}=<[0.7286,0.7286],[0.2355,0.2355],[0.1353,0.1353]>$, \\
& $z_{4}=<[0.7345,0.7345],[0.1353,0.1353],[0.2000,0.2000]>$ \\
\hline \multirow{5}{*}{$I=[0,0.5]$} & $z_{1}=<[0.6643,0.7645],[0.1653,0.2674],[0.1653,0.2493]>$, \\
& $z_{2}=<[0.7345,0.7999],[0.1653,0.2502],[0.1654,0.2503]>$, \\
& $z_{3}=<[0.7286,0.7960],[0.2355,0.2856],[0.1353,0.2174]>$, \\
& $z_{4}=<[0.7345,0.7846],[0.1353,0.2174],[0.2000,0.2674]>$ \\
\hline \multirow{5}{*}{$I=[0,1]$} & $z_{1}=<[0.6643,0.8647],[0.1653,0.3716],[0.1653,0.3345]>$, \\
& $z_{2}=<[0.7345,0.8647],[0.1653,0.3357],[0.1654,0.3383]>$, \\
& $z_{3}=<[0.7286,0.8626],[0.2355,0.3357],[0.1353,0.3000]>$, \\
& $z_{4}=<[0.7345,0.8347],[0.1353,0.3000],[0.2000,0.3357]>$ \\
\hline & $z_{1}=<[0.6643,0.9648],[0.1653,0.4792],[0.1653,0.4214]>$, \\
& $z_{2}=<[0.7345,0.9289],[0.1653,0.4224],[0.1654,0.4314]>$, \\
& $z_{3}=<[0.7286,0.9288],[0.2355,0.3859],[0.1353,0.3834]>$, \\
& $z_{4}=<[0.7345,0.8847],[0.1353,0.3834],[0.2000,0.4055]>$. \\
\hline
\end{tabular}


Table 3. Decision results regarding the SNIEWAA operator

\begin{tabular}{|c|c|c|c|}
\hline$I=\left[I^{-}, I^{+}\right]$ & Score value of $S\left(z_{j}, I\right)$ & Ranking order & The best one \\
\hline$I=[0,0]$ & $0.7858,0.8153,0.7927,0.8047$ & $M_{2}>M_{4}>M_{3}>M_{1}$ & $M_{2}$ \\
\hline$I=[0,0.5]$ & $0.7714,0.7956,0.7806,0.7874$ & $M_{2}>M_{4}>M_{3}>M_{1}$ & $M_{2}$ \\
\hline$I=[0,1]$ & $0.7587,0.7787,0.7698,0.7713$ & $M_{2}>M_{4}>M_{3}>M_{1}$ & $M_{2}$ \\
\hline$I=[0,1.5]$ & $0.7505,0.7725,0.7691,0.7560$ & $M_{2}>M_{3}>M_{4}>M_{1}$ & $M_{2}$ \\
\hline
\end{tabular}

Table 4. Decision results regarding the SNIEWGA operator

\begin{tabular}{|c|c|c|c|}
\hline$I=\left[I^{-}, I^{+}\right]$ & Score value of $S\left(z_{j}, I\right)$ & Ranking order & The best one \\
\hline$I=[0,0]$ & $0.7779,0.8013,0.7859,0.7997$ & $M_{2}>M_{4}>M_{3}>M_{1}$ & $M_{2}$ \\
\hline$I=[0,0.5]$ & $0.7636,0.7839,0.7751,0.7832$ & $M_{2}>M_{4}>M_{3}>M_{1}$ & $M_{2}$ \\
\hline$I=[0,1]$ & $0.7487,0.7657,0.7641,0.7663$ & $M_{4}>M_{2}>M_{3}>M_{1}$ & $M_{4}$ \\
\hline$I=[0,1.5]$ & $0.7330,0.7465,0.7529,0.7492$ & $M_{3}>M_{4}>M_{2}>M_{1}$ & $M_{3}$ \\
\hline
\end{tabular}

However, existing various neutrosophic DM methods cannot handle such a DM problem with the hybrid information of SNS and NN (the SNIS information) and decision makers' indeterminate ranges/cognitions in indeterminate DM applications, while this original study not only can present the SNIS information by describing various indeterminate degrees of the truth, falsity, indeterminacy as a generalization of SNS (SvNS and IvNS), but also can demonstrate the superiority of flexible DM in indeterminate DM applications corresponding to decision makers' indeterminate degrees/cognitions for $I \in\left[I^{-}, I^{+}\right]$. Therefore, this study indicates the convenient and flexible advantages in the indeterminate information expression and processing in indeterminate DM problems.

\section{Conclusions}

This study proposed the SNIS concept for the first time to depict the hybrid information of both SNS and NN in indeterminate and inconsistent setting, and then presented the score, accuracy, and certainty functions of SNIEs for ranking SNIEs and the SNIEWAA and SNIEWGA operators for aggregating SNIEs. Next, a simplified neutrosophic indeterminate multi-attribute DM approach regarding the SNIEWAA and SNIEWGA operators was put forward along with decision makers' indeterminate ranges to deal with indeterminate DM problems in SNIS setting. Eventually, the developed multi-attribute DM approach was applied in an indeterminate DM example on choosing a suitable slope design scheme for an open pit mine in SNIS setting. By the DM example and comparative analysis, we discuss how the different indeterminate ranges affect the ranking orders of alternatives, and then the decision results show the flexibility and effectiveness of the established multi-attribute DM approach in various indeterminate situations of decision makers, which indicate the main superiority in this study. In the future, this study will be further generalized to pattern recognition, medical diagnosis, and image processing in SNIS setting.

\section{Conflicts of Interest}

The authors declare no conflict of interest.

\section{References}

Atanassov, K. (1986). Intuitionistic fuzzy sets. Fuzzy Sets and Systems, 20, 87-96.

https://doi.org/10.1016/S0165-0114(86)80034-3

Atanassov, K., \& Gargov, G. (1989). Interval-valued intuitionistic fuzzy sets. Fuzzy Sets and Systems, 31, 343-349.

https://doi.org/10.1016/0165-0114(89)90205-4

Ali, M., \& Smarandache, F. (2016). Complex neutrosophic set. Neural Computing and Applications, 28, 1817-1834.

https://doi.org/10.1007/s00521-015-2154-y

Ali, M., Deli, I., \& Smarandache, F. (2016). The theory of neutrosophic cubic sets and their applications in pattern recognition. Journal of Intelligent \& Fuzzy Systems, 30(4), 1957-1963. https://doi.org/10.3233/IFS-151906

Alia, M., Son, L. H., Thanhc, N. D., \& Minh, N. V. (2018). A neutrosophic recommender system for medical diagnosis based on algebraic neutrosophic measures. Applied Soft Computing, 71, 1054-1071. https://doi.org/10.1016/j.asoc.2017.10.012

Broumi, S., \& Deli, I. (2014). Correlation measure for neutrosophic refined sets and its application in medical diagnosis. Palestine Journal of Mathematics, 3(1), 11-19.

Broumi, S., \& Smarandache, F. (2015). Interval neutrosophic rough sets. Neutrosophic Sets and Systems, 7, 23-31. https://doi.org/10.1155/2015/232919

Chen, J. Q., Ye, J., \& Du, S. G. (2017). Vector similarity measures between refined simplified neutrosophic sets and their multiple attribute decision making method. Symmetry, 9(8), 153. https://doi.org/10.3390/sym9080153

Can, M. S., \& Ozguven, O. F. (2017). PID tuning with neutrosophic similarity measure. International Journal of Fuzzy Systems, 19(2), 489-503.

https://doi.org/10.1007/s40815-015-0136-y

Gal, A., Vladareanu, L., Smarandache, F., Yu, H., \& Deng, M. (2012). Neutrosophic logic approaches applied to "rabot" real time control. Neutrosophic Theory and Its Applications, $1,55-60$.

Jun, Y. B., Smarandache, F., \& Kim, C. S. (2017). Neutrosophic cubic sets. New Mathematics and Natural Computation, 13(1), 41-54. https://doi.org/10.1142/S1793005717500041 
Köseoğlu, A., Şahin, R., \& Merdan, M. (2019). A simplified neutrosophic multiplicative set-based TODIM using water-filling algorithm for the determination of weights. Expert Systems. https://doi.org/10.1111/exsy.12515

Liu, P. D., \& Wang, Y. M. (2014). Multiple attribute decision making method based on single-valued neutrosophic normalized weighted Bonferroni mean. Neural Computing and Applications, 25(7-8), 2001-2010.

https://doi.org/10.1007/s00521-014-1688-8

Liu, P. D., \& Shi, L. L. (2015). The generalized hybrid weighted average operator based on interval neutrosophic hesitant set and its application to multiple attribute decision making. Neural Computing and Applications, 26(2), 457-471. https://doi.org/10.1007/s00521-014-1736-4

Liu, P. D., \& Liu, X. (2018). The neutrosophic number generalized weighted power averaging operator and its application in multiple attribute group decision making. International Journal of Machine Learning and Cybernetics, 9(2), 347-358. https://doi.org/10.1007/s13042-016-0508-0

Liu, P., Khan, Q., \& Mahmood, T. (2019). Group decision making based on power Heronian aggregation operators under neutrosophic cubic environment. Soft Computing, 24, 19711997. https://doi.org/10.1007/s00500-019-04025-z

Maji, P. K. (2013). Neutrosophic soft set. Annals of Fuzzy Mathematics and Informatics, 5(1), 157-168.

Peng, J. J., Wang, J. Q., Wu, X. H., Wang, J., \& Chen, X. H. (2015). Multivalued neutrosophic sets and power aggregation operators with their applications in multi-criteria group decisionmaking problems. International Journal of Computational Intelligence Systems, 8(2), 345-363.

https://doi.org/10.1080/18756891.2015.1001957

Peng, J. J., Wang, J. Q. Wang, J., Zhang, H. Y., \& Chen, X. H. (2016). Simplified neutrosophic sets and their applications in multi-criteria group decision-making problems. International Journal of Systems Science, 47(10), 2342-2358. https://doi.org/10.1080/00207721.2014.994050

Read, J., \& Stacey, P. (2009). Guidelines for open pit slope design. CSIRO Publishing. https://doi.org/10.1071/9780643101104

Smarandache, F. (1998). Neutrosophy: neutrosophic probability, set, and logic. American Research Press.

Smarandache, F. (2013a). n-Valued refined neutrosophic logic and its applications in physics. Progress in Physics, 4, 143-146.

Smarandache, F. (2013b). Introduction to neutrosophic measure, neutrosophic integral, and neutrosophic probability. Sitech \& Education Publisher.

Smarandache, F. (2014). Introduction to neutrosophic statistics. Sitech \& Education Publishing.

Şahin, R. (2018). Normal neutrosophic multiple attribute decision making based on generalized prioritized aggregation operators. Neural Computing \& Applications, 30(10), 3095-3115. https://doi.org/10.1007/s00521-017-2896-9

Sahin, R. \& Liu, P. D. (2017a). Possibility-induced simplified neutrosophic aggregation operators and their application to multicriteria group decision making. Journal of Experimental \& Theoretical Artificial Intelligence, 29(4), 769-785. https://doi.org/10.1080/0952813X.2016.1259266

Sahin, R. \& Liu, P. D. (2017b). Some approaches to multi criteria decision making based on exponential operations of simplified neutrosophic numbers. Journal of Intelligent \& Fuzzy Systems, 32(3), 2083-2099. https://doi.org/10.3233/JIFS-161695
Thanh, N. D., Ali, M., \& Son, L. H. (2017). A novel clustering algorithm on neutrosophic recommender system for medical diagnosis. Cognitive Computation, 9(4), 526-544.

https://doi.org/10.1007/s12559-017-9462-8

Thong, N. T., Dat, L. Q., Son, L. H., Hoa, N. D., Ali, M., \& Smarandache, F. (2019). Dynamic interval valued neutrosophic set: Modeling decision making in dynamic environments. Computers in Industry, 108, 45-52.

https://doi.org/10.1016/j.compind.2019.02.009

Wang, H., Smarandache, F., Zhang, Y. Q., \& Sunderraman, R. (2005). Interval neutrosophic sets and logic: Theory and applications in computing. Hexis.

Wang, H., Smarandache, F., Zhang, Y. Q., \& Sunderraman, R. (2010). Single valued neutrosophic sets. Multispace and Multistructure, 4, 410-413.

Wu, X. H., Wang, J. Q., Peng, J. J., \& Chen, X. H. (2016). Crossentropy and prioritized aggregation operator with simplified neutrosophic sets and their application in multi-criteria decision-making problems. International Journal of Fuzzy Systems, 18(6), 1104-1116. https://doi.org/10.1007/s40815-016-0180-2

Wu, Q., Wu, P., Zhou, L., Chen, H., \& Guan, X. (2018). Some new Hamacher aggregation operators under single-valued neutrosophic 2-tuple linguistic environment and their applications to multiattribute group decision making. Computers \& Industrial Engineering, 116, 144-162.

https://doi.org/10.1016/j.cie.2017.12.024

Wu, Q., Zhou, L., Chen, Y., \& Chen, H. (2019a). An integrated approach to green supplier selection based on the interval type- 2 fuzzy best-worst and extended VIKOR methods. Information Sciences, 502, 394-417. https://doi.org/10.1016/j.ins.2019.06.049

Wu, Q., Lin, W., Zhou, L., Chen, Y., \& Chen, H. (2019b). Enhancing multiple attribute group decision making flexibility based on information fusion technique and hesitant Pythagorean fuzzy sets. Computers \& Industrial Engineering, 127, 954-970. https://doi.org/10.1016/j.cie.2018.11.029

Ye, J. (2014a). A multicriteria decision-making method using aggregation operators for simplified neutrosophic sets. Journal of Intelligent \& Fuzzy Systems, 26, 2459-2466. https://doi.org/10.3233/IFS-130916

Ye, J. (2014b). Clustering methods using distance-based similarity measures of single-valued neutrosophic sets. Journal of Intelligent Systems, 23(4), 379-389. https://doi.org/10.1515/jisys-2013-0091

Ye, J. (2016). Fault diagnoses of steam turbine using the exponential similarity measure of neutrosophic numbers. Journal of Intelligent \& Fuzzy Systems, 30, 1927-1934. https://doi.org/10.3233/IFS-151903

Ye, J. (2017). Single valued neutrosophic similarity measures based on cotangent function and their application in the fault diagnosis of steam turbine. Soft Computing, 21(3), 817-825. https://doi.org/10.1007/s00500-015-1818-y

Ye, J. (2018). Neutrosophic number linear programming method and its application under neutrosophic number environments. Soft Computing, 22(14), 4639-4646. https://doi.org/10.1007/s00500-017-2646-Z

Ye, S., Fu, J., \& Ye, J. (2015). Medical diagnosis using distancebased similarity measures of single valued neutrosophic multisets. Neutrosophic Sets and Systems, 7, 47-52. 
Ye, J. \& Fu, J. (2016). Multi-period medical diagnosis method using a single valued neutrosophic similarity measure based on tangent function. Computer Methods and Programs in Biomedicine, 123, 142-149.

https://doi.org/10.1016/j.cmpb.2015.10.002

Ye, J., Chen, J. Q., Yong, R., \& Du, S. G. (2017). Expression and analysis of joint roughness coefficient using neutrosophic number functions. Information, 8(2), 69.

https://doi.org/10.3390/info8020069

Yong, R., Ye, J., \& Du, S. G. (2019). A Dice similarity measure for TBM penetrability classification in hard rock condition with the intuitionistic fuzzy information of rock mass properties. European Journal of Environmental and Civil Engineering. https://doi.org/10.1080/19648189.2019.1643789

Zadeh, L. A. (1965). Fuzzy sets. Information and Control, 8, 338353. https://doi.org/10.1016/S0019-9958(65)90241-X

Zhang, H. Y., Wang, J. Q., \& Chen, X. H. (2014). Interval neutrosophic sets and their application in multicriteria decision making problems. The Science World Journal, Article ID 645953. https://doi.org/10.1155/2014/645953

Zhou, L. P., Dong, J. Y., \& Wan, S. P. (2019). Two new approaches for multi-attribute group decision-making with interval-valued neutrosophic Frank aggregation operators and incomplete weights. IEEE Access, 7, 102727-102750.

https://doi.org/10.1109/ACCESS.2019.2927133 